\title{
Nuclear paraspeckle assembly transcript 1 promotes the metastasis and epithelial-mesenchymal transition of hepatoblastoma cells by inhibiting miR-129-5p
}

\author{
MING-CUI FU ${ }^{1 *}$, LI-QUN YUAN ${ }^{2 *}$, TING ZHANG ${ }^{1}$, XIANG-MING YAN $^{1}$, YUN ZHOU $^{1}$, \\ HONG-LIANG XIA ${ }^{1}$, YI WU ${ }^{3}$, LI-XIAO XU ${ }^{4}, \mathrm{XU} \mathrm{CAO}^{1}$ and JIAN WANG ${ }^{1}$ \\ ${ }^{1}$ Department of Surgery, Children's Hospital of Soochow University, Soochow University, Suzhou, \\ Jiangsu 215003; ${ }^{2}$ Department of Neurosurgery, The Second Affiliated Hospital of Soochow University, \\ Soochow University, Suzhou, Jiangsu 215004; ${ }^{3}$ Department of Pathology and ${ }^{4}$ Institute of Pediatrics, \\ Children's Hospital of Soochow University, Soochow University, Suzhou, Jiangsu 215003, P.R. China
}

Received February 22, 2016; Accepted June 27, 2017

DOI: $10.3892 / 01.2017 .6995$

\begin{abstract}
The abnormal expression of nuclear paraspeckle assembly transcript 1 (NEAT1) may serve critical functions for the development and progression of various types of human tumor. However, the expression and biological function of NEAT1 in hepatoblastoma (HB) and the underlying mechanisms for the function of NEAT1 in HB remain largely uncharacterized. In the present study, the results of reverse transcription-quantitative polymerase chain reaction revealed that the expression of NEAT1 was significantly elevated in HB tissues. HB tissues with metastasis also exhibited significantly increased levels of NEAT1 compared with tissues without metastasis. The biological functions of NEAT1 were then assessed using gain-/loss-of-function studies. The results of in vitro assays revealed that inhibiting NEAT1 expression reduced the migration and invasion of HepG2 cells. By contrast, the induced expression of NEAT1 exhibited the opposite effect. The present study also demonstrated that the inhibition of NEAT1 expression prevented the epithelial-mesenchymal transition of HepG2 cells, whereas forced expression of NEAT1 exhibited the opposite effect. In addition, it was confirmed that NEAT1 could modulate the expression of microRNA (miR)-129-5p in HepG2 cells, and that NEAT1 may exert its effect on the metastatic behaviors and epithelial-mesenchymal transition of HepG2 cells by
\end{abstract}

Correspondence to: Dr Xu Cao or Dr Jian Wang, Department of Surgery, Children's Hospital of Soochow University, Soochow University, 303 Jingde Road, Suzhou, Jiangsu 215003, P.R. China

E-mail: cao_xu1982@163.com

E-mail:wj196312@vip.163.com

*Contributed equally

Key words: hepatoblastoma, nuclear paraspeckle assembly transcript 1, metastasis, microRNA-129-5p inhibiting miR-129-5p. In conclusion, the present study indicated that NEAT1 expression was aberrantly increased in $\mathrm{HB}$ and that it may promote the metastasis of HB cells by inhibiting miR-129-5p. Targeting NEAT1 may potentially be a novel therapeutic option for treating patients with HB.

\section{Introduction}

Hepatoblastoma (HB), derived from undifferentiated hepatic progenitor cells (1), is a highly malignant disease in the liver and the most common type of hepatic malignancy in children (2). Local and systemic metastasis are the critical causes for the poor prognosis of this disease $(3,4)$. Exploring the molecular mechanisms underlying the metastatic process of $\mathrm{HB}$ is critical for identifying novel biomarkers and therapeutic targets of HB.

Long non-coding RNAs (lncRNAs) are a group of non-coding RNAs $>200$ nucleotides in length, with a limited ability to code protein (5). However, IncRNAs have been demonstrated to serve important roles in various biological processes, including cell proliferation, differentiation and motility (6). Emerging evidence has demonstrated the aberrant expression of IncRNAs in various types of human cancer $(7,8)$. Deregulated lncRNAs are involved in the growth, drug resistance and metastasis of human cancers (9). However, the pathophysiological functions of lncRNAs in HB remain largely uncharacterized.

Nuclear paraspeckle assembly transcript 1 (NEAT1), an IncRNA occurring in the nucleus that forms the core structural component of the paraspeckle sub-organelles $(10,11)$, was revealed to be abnormally expressed in esophageal squamous cell carcinoma (12), glioma (13), colorectal cancer (14) and prostate cancer (15). The abnormal expression of NEAT1 was significantly associated with the prognosis of patients with cancer (12). However, the expression status, functional role and underlying mechanisms in HB remain unknown.

In the present study, it was identified that the expression of NEAT1 was significantly upregulated in human HB tissues, and the HB tissues with metastasis had significantly increased expression level of NEAT1 compared with those 
without metastasis. Functionally, NEAT1 was revealed to promote the metastatic ability and epithelial-mesenchymal transition (EMT) of HB cells. Furthermore, the present study elucidated that NEAT1 exerts its functional effect in HB cells by inhibiting microRNA (miR)-129-5p.

\section{Materials and methods}

Clinical samples and cell culture. A total of 32 human HB tissue samples and 15 normal liver tissues were obtained from 32 patients with $\mathrm{HB}$ who underwent surgical resection in the Department of Surgery, Children's Hospital of Soochow University (Suzhou, China) between January 2005 and December 2010. The age range of HB patients was between 2 and 9 years old (mean age, 4.6 years), with 20 male and 12 female patients. Clinical specimens were stored at $-80^{\circ} \mathrm{C}$. All samples were obtained subsequent to obtaining written informed consent from every patient. The protocols involving clinical samples were approved by the Ethics Committee of Soochow University, in accordance with the Declaration of Helsinki.

HepG2 HB cells, purchased from American Type Culture Collection (ATCC; Manassas, VA, USA), were cultured in RPMI-1640 medium (Invitrogen; Thermo Fisher Scientific, Inc., Waltham, MA, USA) supplemented with $10 \%$ fetal bovine serum (FBS; Gibco; Thermo Fisher Scientific, Inc.), 100 U/ml penicillin and $100 \mathrm{U} / \mathrm{ml}$ streptomycin. Cells were incubated at $37^{\circ} \mathrm{C}$ in a humidified incubator containing $5 \% \mathrm{CO}_{2}$.

Reverse transcription-quantitative polymerase chain reaction (RT-qPCR). Total RNA was isolated from the HB tissues and HepG2 cells using TRIzol (Thermo Fisher Scientific, Inc.) according to the manufacturer's instructions. To investigate the level of NEAT1 in HB tissues and HepG2 cells, cDNA synthesis was performed using a cDNA reverse transcription kit (Fermentas; Thermo Fisher Scientific, Inc.) according to the manufacturer's instructions, and qPCR for $2 \mathrm{ng}$ cDNA was performed with SYBR-Green I (Takara Biotechnology Co., Ltd., Dalian, China). cDNA was denatured at $95^{\circ} \mathrm{C}$ for $5 \mathrm{~min}$ and subjected to amplification $\left(40\right.$ cycles of $98^{\circ} \mathrm{C}$ for $15 \mathrm{sec}, 58^{\circ} \mathrm{C}$ for $30 \mathrm{sec}$ and $72^{\circ} \mathrm{C}$ for $30 \mathrm{sec}$. The primers used were: NEAT1 forward, 5'-CTTCCTCCCTTTAACTTATCC ATTCAC-3' and reverse, 5'-CTCTTCCTCCACCATTACCAA CAATAC-3'; and $\beta$-actin forward, 5'-AAAGACCTGTAC GCCAACAC-3' and reverse, 5'-GTCATACTCCTGCTTGCT GAT-3'. $\beta$-actin was used as an endogenous control. Relative gene expression was calculated using the $2^{-\Delta \Delta \mathrm{Cq}}$ method (16). Each experiment was repeated at least 3 times.

To measure the relative miR-129-5p level in HepG2 cells, the TaqMan MicroRNA Reverse Transcription kit (Thermo Fisher Scientific, Inc.) was used according to the manufacturer's instructions. qPCR was then performed, using TaqMan miR-129-5p and U6 RNA assays (Thermo Fisher Scientific, Inc.) according to the manufacturer's instructions. Primers for miR-129-5p (cat. no. CD201-0202) and U6 (cat. no. CD201-0145) were obtained from Tiangen Biotech Co., Ltd. (Beijing, China). U6 was used as an endogenous control.

Cell transfection. Small interfering RNAs (siRNAs) against NEAT1 (5'-UGGUAAUGGUGGAGGAAGAUU-3', 5'-GUG
AGA AGUUGCUUAGAAAUU-3'), a scrambled negative control siRNA (cat. no. siN05815122147-1-5), a miR-129-5p inhibitor (cat. no. miR20000242-1-5) and a corresponding negative control vector (cat. no. NC-miR20000242-1-5) were purchased from Guangzhou RiboBio Co., Ltd. (Guangzhou, China). A NEAT1-expressing vector and a control vector were purchased from Invitrogen (Thermo Fisher Scientific, Inc.). The aforementioned vectors were transfected into HepG2 cells with Lipofectamine $2000{ }^{\circledR}$ (Invitrogen; Thermo Fisher Scientific, Inc.) according to the manufacturer's instructions.

Transwell assay. The migration and invasion ability of HepG2 cells was measured with a Transwell assay. A total of $2 \times 10^{3}$ HepG2 cells resuspended in serum-free medium were seeded into Transwell inserts with $8-\mu \mathrm{m}$ pores (EMD Millipore, Billerica, MA, USA), whereas the lower chamber was filled with $600 \mu \mathrm{l}$ medium containing $10 \% \mathrm{FBS}$ as an attractant. For the invasion assay, the upper chamber was coated with a mixture of RPMI-1640 medium and Matrigel (BD Biosciences, Franklin Lakes, NJ, USA) at a ratio of 7:1. Cells were then incubated in the previously described conditions $\left(37^{\circ} \mathrm{C}\right.$ in a humidified incubator containing $5 \% \mathrm{CO}_{2}$ ). At $36 \mathrm{~h}$ after seeding, the migrated or invaded HepG 2 cells on the lower surface were stained with $0.1 \%$ crystal violet for $5 \mathrm{~min}$ at room temperature, and cell numbers were counted from 10 random fields of the lower surface of the filter using a light microscope at x20 magnification.

Western blot analysis. Radioimmunoprecipitation assay lysis buffer (Beyotime Institute of Biotechnology, Shanghai, China) was used to extract protein from HepG2 cells, and protein concentration was measured using a bicinchoninic acid kit (Pierce; Thermo Fisher Scientific, Inc.), according to the manufacturer's instructions. A total of $40 \mu \mathrm{g}$ of protein from each sample was separated on a Novex ${ }^{\circledR} 10 \%$ Tris-glycine gel, then transferred to a nitrocellulose membrane (both from Thermo Fisher Scientific, Inc.). The membrane was blocked with $10 \%$ goat serum (BD Biosciences) at room temperature for $30 \mathrm{~min}$. The membranes were incubated with the following primary antibodies overnight at $4^{\circ} \mathrm{C}$ : E-cadherin (1:1,000 dilution, cat. no. 3195; Cell Signaling Technologies, Inc., Danvers, MA, USA), vimentin (1:1,000 dilution, cat. no. sc-5565) and GAPDH (1:1,500 dilution, cat. no. sc-32233; Santa Cruz Biotechnology, Inc., Dallas, TX, USA). The membranes were then incubated with anti-mouse (cat. no. 32430) or anti-rabbit (cat. no. 65-6120) secondary antibodies (1:10,000 dilution; Pierce; Thermo Fisher Scientific, Inc.) at room temperature for $1 \mathrm{~h}$, and immunoreactive signals were detected using the Bio-Rad Gel imaging system (Bio-Rad Laboratories, Inc., Hercules, CA, USA). Quantifaction of immunoblots images were performed using Image Lab software (version 6.0; Bio-Rad Laboratories, Inc.).

Prediction of NEAT1 targets. The prediction of the target miRNAs of NEAT1 was performed using starBase (http://starbase.sysu.edu.cn/), a publicly available database of miRNAs.

Statistical analysis. All quantitative data are expressed as the mean \pm standard deviation. GraphPad Prism 5 software (GraphPad Software, Inc., La Jolla, CA, USA) was used to 

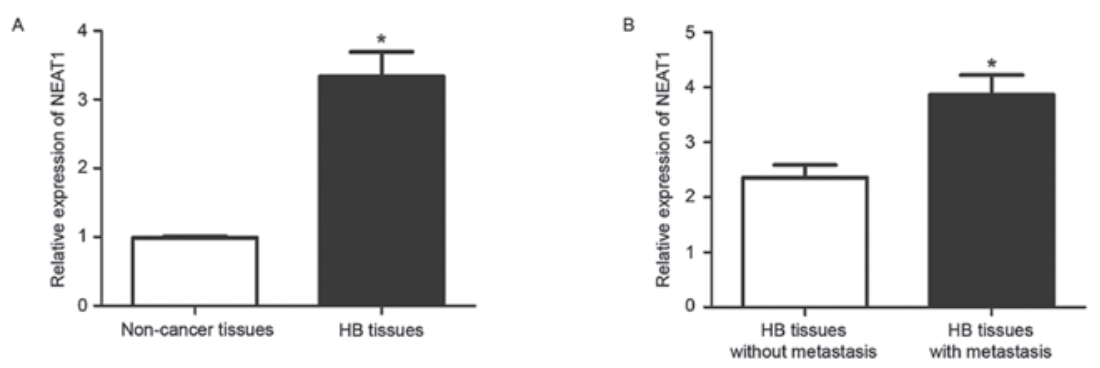

Figure 1. Expression status of NEAT1 in HB and non-cancer tissue. Differences in NEAT1 expression between (A) HB tissues and normal tissues, and (B) HB tissues with and without metastasis. ${ }^{*} \mathrm{P}<0.05$. HB, hepatoblastoma; NEAT1, nuclear paraspeckle assembly transcript 1.
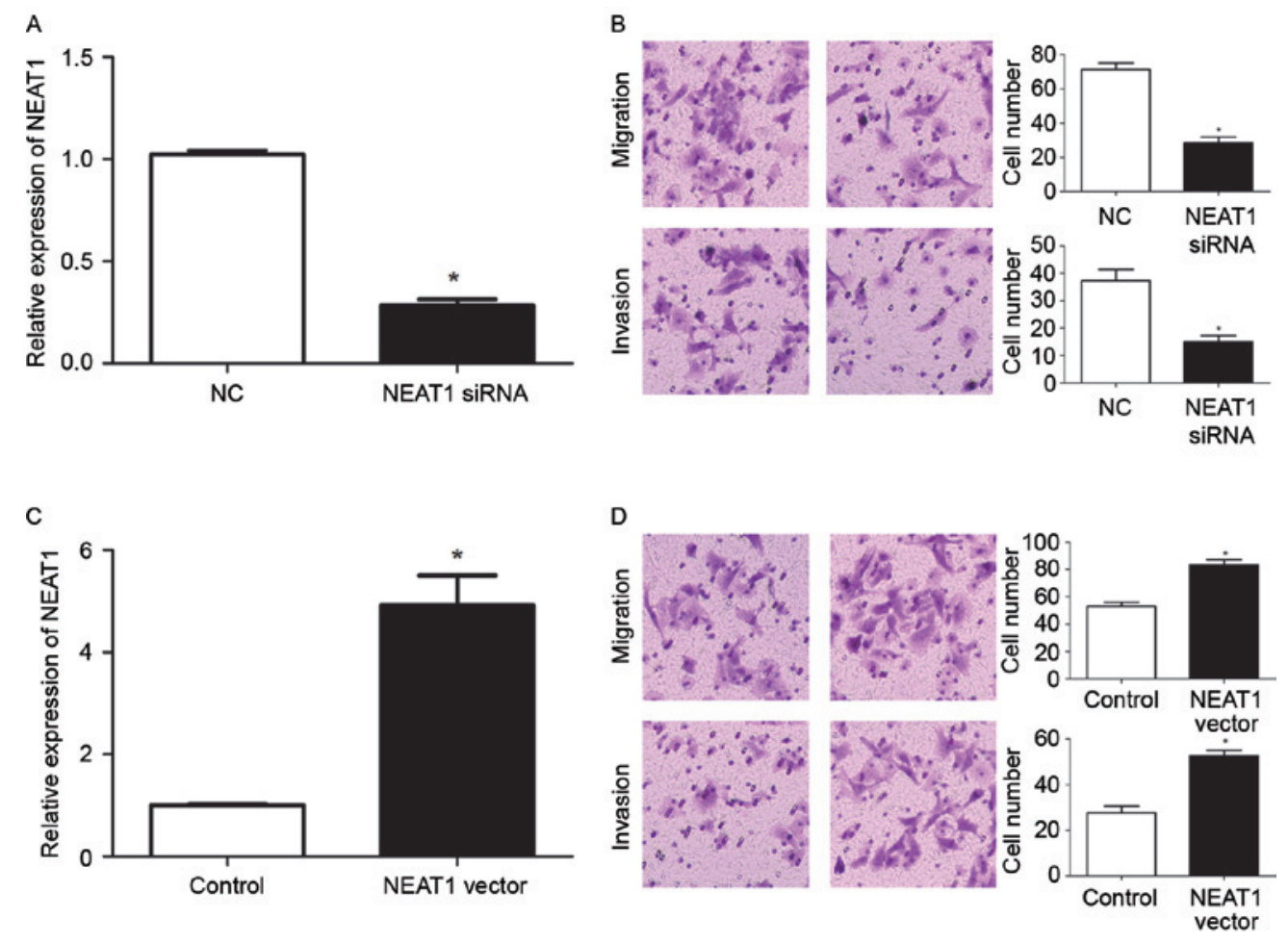

Figure 2. NEAT1 promotes the migration and invasion of HepG2 cells. (A) Transfection of NEAT1 siRNA significantly decreased NEAT1 expression in HepG2 cells. (B) Inhibition of NEAT1 significantly reduced the migration and invasion of HepG2 cells. Magnification, $\mathrm{x} 20 ; \mathrm{n}=3$ repeats with similar results. (C) NEAT1-expressing vector significantly increased the level of NEAT1 in HepG2 cells. (D) Overexpression of NEAT1 significantly increased the migration and invasion of 5-8F cells. Magnification, $\mathrm{x} 20 ; \mathrm{n}=3$. " $\mathrm{P}<0.05$. NEAT1, nuclear paraspeckle assembly transcript 1 ; siRNA, small interfering RNA; NC, negative control.

perform statistical analysis. Pearson's $\chi^{2}$ test (for enumerative data) and Student's t-test (for quantitative data) were performed in the present study. $\mathrm{P}<0.05$ was considered to indicate a statistically significant difference.

\section{Results}

NEATl expression is significantly upregulated in HB tissues. NEAT1 expression levels in $32 \mathrm{HB}$ tissues and 15 non-tumor tissues were measured by RT-qPCR. The expression level of NEAT1 in HB tissues was significantly increased compared with non-tumor tissues $(\mathrm{P}<0.05$; Fig. 1A). The expression of NEAT1 was then compared between HB tissues from patients with and without metastasis. The results of RT-qPCR revealed that HB tissues with metastasis had significantly increased levels of NEAT1 compared with those without metastasis $(\mathrm{P}<0.05$; Fig. 1B). These data indicated that NEAT1 performs an oncogenic role in $\mathrm{HB}$ and possibly contributes to the metastasis of $\mathrm{HB}$.

NEAT1 promotes the migration and invasion of HepG2 cells. Subsequent to confirming the aberrant expression of NEAT1 in HB tissues, the functional effects of NEAT1 in HepG2 cells were examined. Since HB tissues with metastasis had significantly increased levels of NEAT1, it was investigated whether NEAT1 could promote the migration and invasion of HepG2 cells. NEAT1-specific siRNA was used to inhibit the expression of NEAT1 in HepG2 cells. Transfection of NEAT1 siRNA significantly reduced the expression level of NEAT1 in HepG2 cells $(\mathrm{P}<0.05$; Fig. 2A). Transwell assays were then used to investigate the alteration of the migration and invasion of HepG2 cells subsequent to inhibiting the expression of NEAT1. Transwell assays revealed that the downregulation of NEAT1 expression in HepG2 cells significantly reduced 

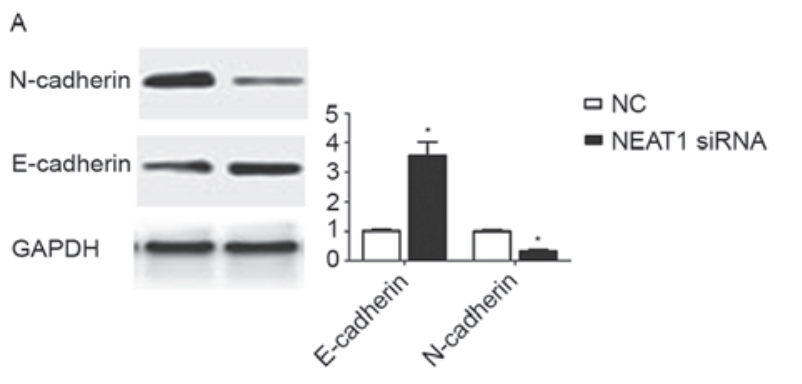

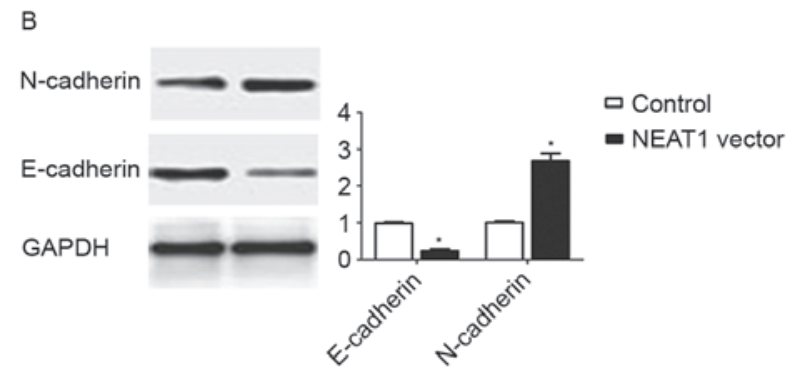

Figure 3. NEAT1 promotes the epithelial-mesenchymal transition of HepG2 cells. (A) Inhibition of NEAT1 significantly increased E-cadherin expression and decreased N-cadherin expression in HepG2 cells. (B) Overexpression of NEAT1 decreased E-cadherin expression and increased N-cadherin expression in HepG2 cells. ${ }^{*} \mathrm{P}<0.05$. NEAT1, nuclear paraspeckle assembly transcript 1; siRNA, small interfering RNA; NC, negative control.
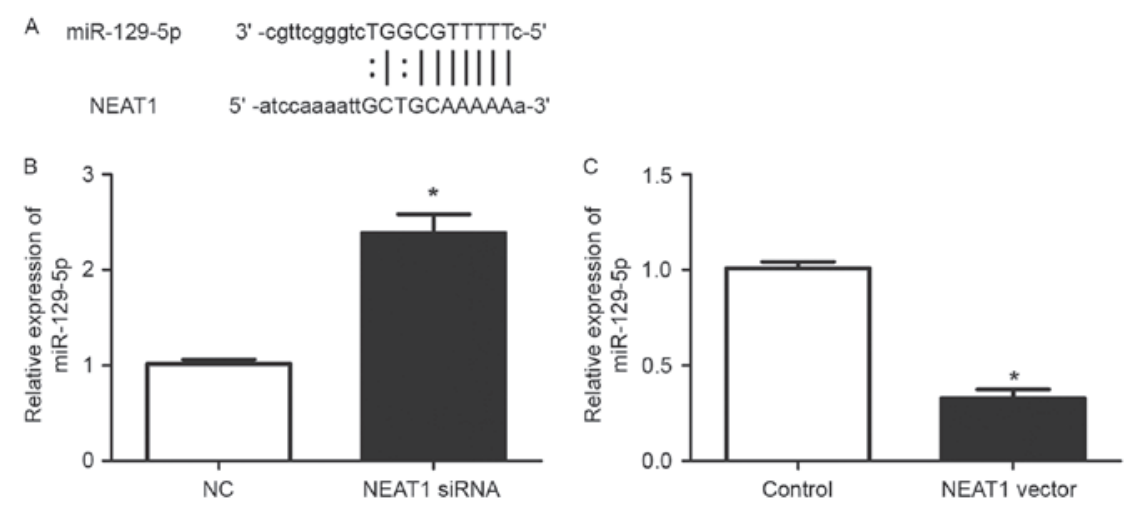

Figure 4. NEAT1 inhibits the expression of miR-129-5p in HepG2 cells. (A) NEAT1 contains a complementary sequence for miR-129-5p. (B) Inhibition of NEAT1 significantly increased the expression level of miR-129-5p in HepG2 cells. (C) Forced expression of NEAT1 significantly decreased the expression level of miR-129-5p in HepG2 cells. "P<0.05. NEAT1, nuclear paraspeckle assembly transcript 1; siRNA, small interfering RNA; NC, negative control.

the migration and invasion of HepG2 cells $(\mathrm{P}<0.05$; Fig. $2 \mathrm{~B})$. To confirm the modulatory function of NEAT1 in the metastatic ability of HepG2 cells, a NEAT1-expressing vector was used to overexpress NEAT1 in HepG2 cells. Transfection of the NEAT1 vector significantly increased the expression of NEAT1 in HepG2 cells ( $\mathrm{P}<0.05$; Fig. $2 \mathrm{C}$ ), and resulted in a significantly increased extent of migration and invasion of HepG2 cells $(\mathrm{P}<0.05$; Fig. 2D). Taken together, these data indicated that NEAT1 may enhance the metastatic ability of HepG2 cells.

NEATl contributes to the EMT of HepG2 cells. During the last two decades, EMT, which was characterized as decreased expression of epithelial marker (E-cadherin) and increased expression of mesenchymal marker ( $\mathrm{N}$-cadherin), was recognized as an important mechanism in cancer metastasis (17). Since the data of the present study confirmed the enhancing effect of NEAT1 on the metastatic ability of HepG2 cells, it was subsequently explored whether NEAT1 expression could promote the EMT of HepG2 cells. The results of western blotting revealed that inhibition of NEAT1 in HepG2 cells resulted in significantly increased E-cadherin expression $(\mathrm{P}<0.05$; Fig. 3A) and decreased $\mathrm{N}$-cadherin expression $(\mathrm{P}<0.05$; Fig. 3A). By contrast, overexpression of NEAT1 in HepG2 cells led to the decreased expression of E-cadherin $(\mathrm{P}<0.05$; Fig. 3B) and the increased expression of $\mathrm{N}$-cadherin $(\mathrm{P}<0.05$; Fig. 3B). These data indicated that the expression of NEAT1 may promote EMT in HepG2 cells.
Expression level of miR-129-5p in HepG2 cells is modulated by NEAT1. It has been demonstrated that IncRNAs contain complementary sequences to miRNAs and may inhibit the expression and activity of miRNAs (18). To explore whether NEAT1 has similar mechanisms in HepG2 cells, the prediction of microRNA target sites was performed with the starBase database. Among numerous potential microRNA targets, the present study focused on miR-129-5p, as it had previously been revealed to serve an important role in modulating the metastasis and EMT of human cancer (19-21). As demonstrated in Fig. 4A, NEAT1 contained a complementary sequence against miR-129-5p. RT-qPCR was then used to confirm whether NEAT1 affects the expression of miR-129-5p in HepG2 cells. The results of RT-qPCR demonstrated that knockdown of NEAT1 significantly increased the expression of miR-129-5p $(\mathrm{P}<0.05$; Fig. 4B), while overexpression of NEAT1 significantly reduced miR-129-5p level in HepG2 cells $(\mathrm{P}<0.05$; Fig. 4C). These results demonstrated that NEAT1 may inhibit the expression of miR-129-5p in HepG2 cells.

NEAT1 exerts its functional effects on the metastasis and EMT of HepG2 cells through modulating miR-129-5p. To confirm that NEAT1 exerts its functional effect in HepG2 cells by modulating miR-129-5p, HepG2 cells transfected with NEAT1 siRNA were then transfected with an miR-129-5p inhibitor. Transfection with the miR-129-5p inhibitor significantly reduced the miR-129-5p expression of HepG2 cells $(\mathrm{P}<0.05$; Fig. 5A). Functionally, the inhibition of miR-129-5p in HepG2 

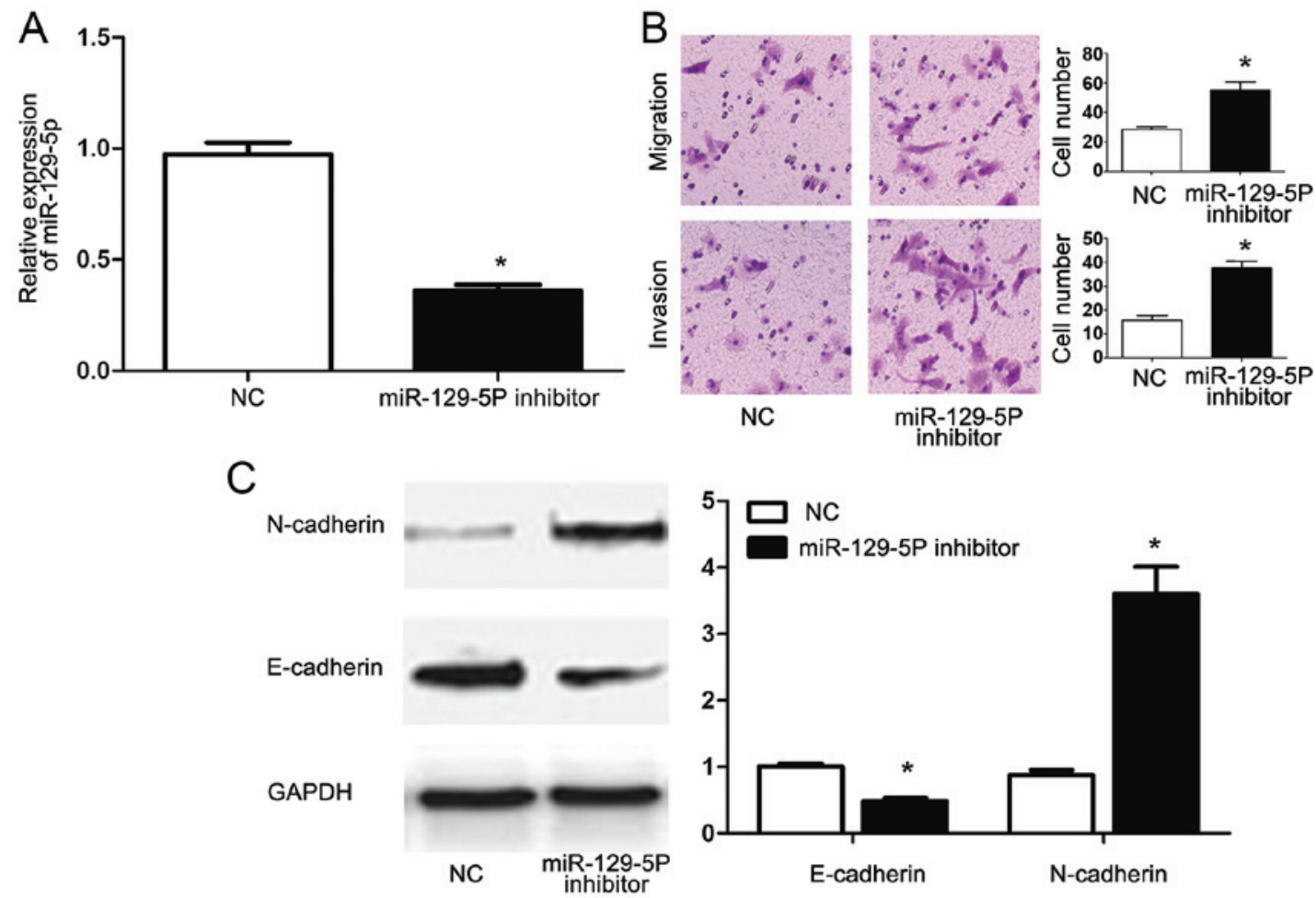

Figure 5. NEAT1 exerts its functional effects on HepG2 cells by inhibiting miR-129-5p cells. (A) miR-129-5p inhibitor significantly reduced the expression level of miR-129-5p in HepG2 cells transfected with NEAT1 siRNA. (B) Inhibition of miR-129-5p reversed the inhibitory effects of NEAT1 knockdown on the migration and invasion of HepG2 cells. Magnification, x20. (C) Inhibition of miR-129-5p abrogated the inhibitory effects of NEAT1 knockdown on the epithelial-mesenchymal transition of HepG2 cells. $\mathrm{n}=3$. ${ }^{*} \mathrm{P}<0.05$. NEAT1, nuclear paraspeckle assembly transcript 1; NC, negative control.

cells transfected with NEAT1 siRNA reversed the inhibitory effects of NEAT1 siRNA on the migration and invasion of HepG2 cells. Furthermore, inhibition of miR-129-5p decreased E-cadherin $(\mathrm{P}<0.05$; Fig. 5B) and increased $\mathrm{N}$-cadherin $(\mathrm{P}<0.05$; Fig. 5B) expression in HepG2 cells transfected with NEAT1 siRNA, indicating that miR-129-5p inhibition reversed the inhibitory effects of NEAT1-knockdown on EMT of HepG2 cells. These data indicated that NEAT1 may not only modulate the expression of miR-129-5p, but also exert its functional effects on metastasis and EMT of HepG2 cells through modulating miR-129-5p expression.

\section{Discussion}

The metastasis of HB is the major reason for the poor prognosis of patients with HB (1-4). There are currently few effective treatments available for patients with metastasis. lncRNAs have been identified to serve an important role in cancer development and progression $(12,15)$. Exploring the expression status and biological functions of lncRNAs in HB may contribute to the identification of novel biomarkers and therapeutic targets for patients with HB.

Among a number of cancer-associated lncRNAs, NEAT1 has been identified to be an oncogenic lncRNA; it may be overexpressed in prostate cancer (22), colorectal cancer (20), glioma (13) and esophageal squamous cell carcinoma (12), and its overexpression was associated with poor prognosis of the patients with cancer (12). In the present study, it was revealed that NEAT1 was overexpressed in HB tissues. Notably, tumor tissues from patients with metastasis were found to have significantly increased expression levels of NEAT1 compared with patients without metastasis, indicating its oncogenic function and potential ability to promote metastasis in HB.

Previous studies have demonstrated that NEAT1 has various biological functions in various types of human cancer. For example, a previous study on prostate cancer revealed that NEAT1 may promote the growth of prostate cancer by affecting the epigenetic status of the target gene promoter (15). An additional study on lung cancer demonstrated that NEAT1 was associated with the apoptosis and cell cycle progression modulated by miR-449a (23). NEAT1 has also been reported to serve a role in the regulation of the pro-tumorigenic hypoxia phenotype in breast cancer (24). Therefore, these previous studies demonstrated that the biological role of NEAT1 in human cancers varies between cancer types. In the present study, the results of Transwell assays demonstrated that the knockdown of NEAT1 inhibits the migration and invasion of HB cells, while overexpression of NEAT1 promotes the migration and invasion of HB cells. In addition, the results of western blotting revealed that NEAT1 may promote the EMT of HepG2 cells. Therefore, the results of the present study demonstrated that NEAT1 promoted the metastatic ability and EMT of HB cells.

lncRNAs may act as a 'sponge' for miRNAs and regulate the expression and function of miRNAs $(22,25,26)$, thus affecting the development and progression of various types of human cancer. Therefore, it was investigated whether NEAT1 may exert its functional effects on HB through similar mechanisms. Using starBase, NEAT1 was revealed to contain a complementary sequence of miR-129-5p, indicating that NEAT1 may regulate the expression of miR-129-5p. RT-qPCR was then used to 
confirm whether NEAT1 regulates the expression of miR-129-5p in HepG2 cells. The results of RT-qPCR revealed that the knockdown of NEAT1 increased the level of miR-129-5p, while overexpression of NEAT1 decreased the level of miR-129-5p in HepG 2 cells. These results demonstrated that NEAT1 may inhibit the expression of miR-129-5p in HepG2 cells.

Since previous studies have confirmed that miR-129-5p regulates the metastasis and EMT of cancer cells (19-21), the present study investigated whether NEAT1 exerted its functional effects on HepG2 cells through modulating the expression of miR-129-5p. An miR-129-5p inhibitor was used to inhibit the expression level of miR-129-5p in HepG2 cells transfected with NEAT1 siRNA. The results of Transwell assays revealed that inhibiting the expression of miR-129-5p partially reversed the inhibitory effects of NEAT1 knockdown on cell migration and invasion. Furthermore, the western blotting results demonstrated that inhibiting miR-129-5p may abrogate the inhibitory effects of NEAT1 knockdown on EMT. These results indicated that NEAT1 may not only inhibit the expression of NEAT1 in HepG2 cells, but also exert its functional effects on HepG2 cells by modulating the expression of miR-129-5p.

In conclusion, to the best of our knowledge, the present study demonstrated for the first time that the expression of NEAT1 is significantly elevated in HB tissues compared with non-cancerous liver tissue. HB tissue from patients with metastasis exhibited a significantly increased level of NEAT1 expression relative to $\mathrm{HB}$ tissue from patients that did not exhibit metastasis. NEAT1 may promote the EMT and metastatic behaviors of HB cells. Furthermore, NEAT1 may inhibit miR-129-5p, and the functional effects of NEAT1 in HB may be mediated by modulating miR-129-5p expression.

\section{Acknowledgements}

The present study was supported by the Natural Science Foundation of China (grant no. 81502496), the Science Foundation of Jiangsu Province Health Department (grant nos. Q201503 and Q201304), Planning Project of Science and Technology Development of Suzhou (grant no. SYS201763) and the Jiangsu Government Scholarship for Overseas Studies.

\section{References}

1. Weinberg AG and Finegold MJ: Primary hepatic tumors of childhood. Hum Pathol 14: 512-537, 1983.

2. Ishak KG and Glunz PR: Hepatoblastoma and hepatocarcinoma in infancy and childhood. Report of 47 cases. Cancer 20: 396-422, 1967.

3. Czauderna P, Otte JB, Roebuck DJ, von Schweinitz D and Plaschkes J: Surgical treatment of hepatoblastoma in children. Pediatr Radiol 36: 187-191, 2006.

4. Haas JE, Muczynski KA, Krailo M, Ablin A, Land V, Vietti TJ and Hammond GD: Histopathology and prognosis in childhood hepatoblastoma and hepatocarcinoma. Cancer 64: 1082-1095, 1989.

5. Mercer TR, Dinger ME and Mattick JS: Long non-coding RNAs: Insights into functions. Nat Rev Genet 10: 155-159, 2009.
6. Takahashi K, Yan I, Haga H and Patel T: Long noncoding RNA in liver diseases. Hepatology 60: 744-753, 2014.

7. Zhang H, Chen Z, Wang X, Huang Z, He Z and Chen Y: Long non-coding RNA: A new player in cancer. J Hematol Oncol 6: 37, 2013.

8. Prensner JR and Chinnaiyan AM: The emergence of lncRNAs in cancer biology. Cancer Discov 1: 391-407, 2011.

9. Tsai MC, Spitale RC and Chang HY: Long intergenic noncoding RNAs: New links in cancer progression. Cancer Res 71: 3-7, 2011.

10. Clemson CM, Hutchinson JN, Sara SA, Ensminger AW, Fox AH, Chess A and Lawrence JB: An architectural role for a nuclear noncoding RNA: NEAT1 RNA is essential for the structure of paraspeckles. Mol Cell 33: 717-726, 2009.

11. Souquere S, Beauclair G, Harper F, Fox A and Pierron G: Highly ordered spatial organization of the structural long noncoding NEAT1 RNAs within paraspeckle nuclear bodies. Mol Biol Cell 21: 4020-4027, 2010

12. Chen X, Kong J, Ma Z, Gao S and Feng X: Up regulation of the long non-coding RNA NEAT1 promotes esophageal squamous cell carcinoma cell progression and correlates with poor prognosis. Am J Cancer Res 5: 2808-2815, 2015.

13. Zhen L, Yun-Hui L, Hong-Yu D, Jun M and Yi-Long Y: Long noncoding RNA NEAT1 promotes glioma pathogenesis by regulating miR-449b-5p/c-Met axis. Tumour Biol 37: 673-683, 2016.

14. Li Y, Li Y, Chen W, He F, Tan Z, Zheng J, Wang W, Zhao Q and Li J: NEAT expression is associated with tumor recurrence and unfavorable prognosis in colorectal cancer. Oncotarget 6: 27641-27650, 2015.

15. Chakravarty D, Sboner A, Nair SS, Giannopoulou E, Li R, Hennig S, Mosquera JM, Pauwels J, Park K, Kossai M, et al: The oestrogen receptor alpha-regulated lncRNA NEAT1 is a critical modulator of prostate cancer. Nat Commun 5: 5383, 2014.

16. Livak KJ and Schmittgen TD: Analysis of relative gene expression data using real-time quantitative PCR and the 2(-Delta Delta C(T)) method. Methods 25: 402-408, 2001.

17. Yilmaz M and Christofori G: EMT, the cytoskeleton, and cancer cell invasion. Cancer Metastasis Rev 28: 15-33, 2009.

18. Jalali S, Bhartiya D, Lalwani MK, Sivasubbu S and Scaria V: Systematic transcriptome wide analysis of lncRNA-miRNA interactions. PLoS One 8: e53823, 2013.

19. Li M, Tian L, Wang L, Yao H, Zhang J, Lu J, Sun Y, Gao X, Xiao H and Liu M: Down-regulation of miR-129-5p inhibits growth and induces apoptosis in laryngeal squamous cell carcinoma by targeting APC. PLoS One 8: e77829, 2013.

20. Liu Y, Hei Y, Shu Q, Dong J, Gao Y, Fu H, Zheng X and Yang G: $\mathrm{VCP} / \mathrm{p} 97$, down-regulated by microRNA-129-5p, could regulate the progression of hepatocellular carcinoma. PLoS One 7 : e35800, 2012.

21. Duan L, Hao X, Liu Z, Zhang Y and Zhang G: miR-129-5p is down-regulated and involved in the growth, apoptosis and migration of medullary thyroid carcinoma cells through targeting RET. FEBS Lett 588: 1644-1651, 2014.

22. Salmena L, Poliseno L, Tay Y, Kats L and Pandolfi PP: A ceRNA hypothesis: The rosetta stone of a hidden RNA language? Cell 146: 353-358, 2011.

23. You J, Zhang Y, Liu B, Li Y, Fang N, Zu L, Li X and Zhou Q: MicroRNA-449a inhibits cell growth in lung cancer and regulates long noncoding RNA nuclear enriched abundant transcript. Indian J Cancer 51 (Suppl 3): e77-e81, 2014.

24. Choudhry H, Albukhari A, Morotti M, Haider S, Moralli D, Smythies J, Schödel J, Green CM, Camps C, Buffa F, et al: Tumor hypoxia induces nuclear paraspeckle formation through HIF-2 $\alpha$ dependent transcriptional activation of NEAT1 leading to cancer cell survival. Oncogene 34: 4482-4490, 2015.

25. Sumazin P, Yang X, Chiu HS, Chung WJ, Iyer A, Llobet-Navas D, Rajbhandari P, Bansal M, Guarnieri P, Silva J and Califano A: An extensive microRNA-mediated network of RNA-RNA interactions regulates established oncogenic pathways in glioblastoma. Cell 147: 370-381, 2011

26. Deng L, Yang SB, Xu FF and Zhang JH: Long noncoding RNA CCAT1 promotes hepatocellular carcinoma progression by functioning as let-7 sponge. J Exp Clin Cancer Res 34: 18, 2015. 